\title{
An intelligent algorithm to recommend percent vegetation cover (ARVC) for $\mathrm{PM}_{2.5}$ reduction
}

- Amir Masoud Rahmani,

- Seyedeh Yasaman Hosseini Mirmahaleh \&

- Mehdi Hosseinzadeh

Air Quality, Atmosphere \& Health (2020)

Abstract

Nowadays, increasing particulate matter (PM) remains a challenge for environmental and humanity health and increases death statistics. Case studies and experimental observations demonstrated that vegetation coverage and type of plants affect PM and air quality. Condensation of $\mathrm{PM}_{2.5}$ has an impressive effect on deteriorating air quality. Increasing vegetation coverage has a significant impact on reducing $\mathrm{PM}_{2.5}$. However, the requirement percent vegetation cover (PVC) is likewise a shadow for careful analysis to recommend the requirement percent and area of vegetation for different parts of the metropolitan. In this paper, we propose a four-phase intelligent algorithm for investigating $\mathrm{PM}_{2.5}$ and critical situations to detect unhealthy air quality monitoring stations (AQMSs). Our algorithm makes a decision based on fuzzy and neural network methods and recommends the percent density and area of vegetation. Our analysis of the weather condition is event-driven, considering rainfall as an event to examine the situation of each AQMS before and after rainfall. The experiments demonstrate reducing $\mathrm{PM}_{2.5}>150$ to $\mathrm{PM}_{2.5}<50$ using recommending PVC of approximately $20-74 \%$. We achieved these results by periodically estimating and evaluating weather conditions in the autumn and winter as two critical seasons of the year.

- DOlhttps://doi.org/10.1007/s11869-020-00844-4

Keywords

- Vegetation density

- Air quality monitoring station (AQMS)

- Percent vegetation cover (PVC) fuzzy system 
- Recommender algorithm

- Neural network 